\title{
Efficacy of a four-week uphill sprint training intervention in field hockey players
}

John R. Jakeman, Judith McMullan, John A. Babraj

This is a non-final version of an article published in final form in Journal of Strength \& Conditioning Research.

Jakeman, J.R., McMullan, J. and Babraj, J.A. 2016. Efficacy of a four-week uphill sprint training intervention in field hockey players. Journal of Strength \& Conditioning Research. doi: 10.1519/JSC.0000000000001373 
Efficacy of a four-week uphill sprint training intervention in field hockey players John R. Jakeman*1, Judith McMullan², John A. Babraj ${ }^{3}$

Affiliations: ${ }^{1}$ Department of Health and Life Sciences, Oxford Brookes University; ${ }^{2 .}$

Dundee High School; ${ }^{3 .}$ Division of Sport and Exercise Science, Abertay University,

Dundee

Corresponding author: *Dr, John Jakeman,

Faculty of Health and Life Sciences,

Department of Sport and Health Sciences,

Oxford Brookes University

OX3 OBP

Tel: 01865483815

Fax: 01865483591

Email: jiakeman@brookes.ac.uk

Running head: High intensity training in Hockey 


\section{Abstract}

Current evidence increasingly suggests that very short, supra-maximal bouts of exercise can have significant health and performance benefits. The majority of research conducted in the area however, uses laboratory-based protocols, which can lack ecological validity. The purpose of this study was to examine the effects of a high intensity sprint-training programme on hockey related performance measures. 14 semiprofessional hockey players completed either a 4-week high intensity training (HIT) intervention, consisting of a total of six sessions HIT, which progressively increased in volume $(n=7)$, or followed their normal training programme (Con; $n=7)$. Straight-line sprint speed with and without a hockey stick and ball, and slalom sprint speed, with and without a hockey stick and ball were used as performance indicators. Maximal sprint speed over $22.9 \mathrm{~m}$ was also assessed. Upon completion of the four-week intervention, straight-line sprint speed improved significantly in the HIT group ( 3\%), with no change in performance for the Con group. Slalom sprint speed, both with and without a hockey ball was not significantly different following the training programme in either group. Maximal sprint speed improved significantly (12.1\%) in the HIT group, but there was no significant performance change in the Con group. The findings of this study indicate that a short period of HIT can significantly improve hockey related performance measures, and could be beneficial to athletes and coaches in field settings. 
Key words:

Uphill sprinting; Team Sport; sprint performance INTRODUCTION

While a wide range of training approaches are used in competitive team sports, further research is required to elucidate the optimal training methods for specific sports (27). One of the principles of training, which is often cited as necessary for performance improvement is that of specificity (10). Field hockey, soccer and rugby union all have similar time motion characteristics, with large proportions of time being spent either walking or running (46.5 and $40.5 \%$ respectively for field hockey (27)). As such the specificity principle would suggest that walking and running should be the main focus of athletic conditioning. However traditional training methods for aerobic fitness have typically utilized long duration exercise (13), and given that, the ability to generate a high maximal speed on repeated occasions is beneficial to performance within team sports (27), well-constructed training programmes often include aspects of repeated sprint exercise to facilitate developments in muscular strength, power and endurance (2).

There is a growing body of evidence indicating that very high intensity, maximal effort repeated sprinting can not only be beneficial for improvements in sprint performance, but can also elicit physiological adaptations more commonly associated with prolonged bouts of relatively low intensity exercise training $(12,17,19,21)$. A variety of exercise 
protocols have been developed using this approach, ranging from a very short, 6 second sprint, with a one minute recovery period repeated ten times (17) to a longer 30 second sprint, with a four minute recovery, repeated four or more times (4). All of these approaches have been shown to elicit similar performance improvements in both power and endurance parameters. In team sports, HIT has been shown to improve maximal aerobic speed, increase anaerobic work capacity and lower $40 \mathrm{~m}$ sprint time $(9,28)$.

While there is an increasingly convincing body of evidence to support the use of this training approach, the majority of studies have used relatively expensive laboratory based ergometers. Although this approach allows robust control of the exercise intensity, it does not necessarily possess a practicality, which would make it an applicable conditioning tool. The purpose of this study therefore, was to assess the efficacy of an uphill running HIT programme for field hockey performance.

\section{METHODS}

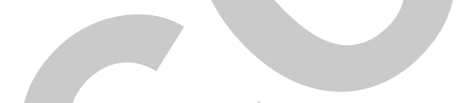

\section{Experimental approach to the problem}

In this study, a randomized experimental design was used to investigate the efficacy of the training modality, using a pre-test post-test model. Before and after two weeks of either an exercise intervention, or following a normal training programme, participants 
completed a range of performance assessments, with data being analysed to determine whether there had been an effect of the intervention.

Subjects

Fourteen semi-professional female field hockey players (18-26 years) were recruited to participate in the study, which received ethical approval from the local ethics committee. All participants were advised of the benefits and risks associated with the study, and provided written informed consent prior to commencement. All participants played hockey for approximately 5 hours per week, and regularly completed two non-hockey specific training sessions per week. Data were collected over a 6 week period, and participants were randomly allocated into a control or treatment group $(n=7)$, with the control group maintaining their normal training routine, and the treatment group replacing the two non-hockey specific sessions with two HIT sessions. Pre and posttest data were collected in week 1 and week 6 , and during weeks 2-5 HIT was completed by participants in the treatment group.

Procedures

Baseline assessments of performance were completed on two occasions, prior to normal hockey training sessions. During the first session, a shuttle sprint dribble test (SDT) was completed to establish baseline performance. In this case, $6 \times 32 \mathrm{~m}$ shuttles were completed while carrying a hockey stick, with a 30 second rest between each shuttle. After a 5-minute recovery the same test was repeated, while dribbling a hockey ball. 
Five minutes after the SDT, a Slalom SDT (fig. 1), was completed 3 times with a minutes rest between each set. After a 5 minute recovery the same test was repeated but while dribbling a hockey ball. These assessments followed the protocol of Lemmink et al. (20), who reported intraclass correlation coefficients of $0.81,0.91$ for shuttle sprint and dribble respectively, and 0.91 and 0.78 for slalom sprint and dribble respectively.

Insert figure 1 here

Figure 1- Slalom sprint test and dribble test (Slalom STD), (20).

In a second session, a running repeated sprint ability test was completed, which consisted of sprinting $22.9 \mathrm{~m}$ (quarter of a synthetic hockey pitch) at maximal rate (100\%). 6 sprint repetitions were performed with a 30 second rest between each sprint. Sprint times were recorded using infra-red light gates (Brower, USA).

\section{Training protocol}

Participants in the treatment group completed a progressively increasing HIT twice per week over a period of four weeks. All sprints were completed outdoors up a vertical incline of $8 \%$. Both sessions of each training week were identical, which in training week 1 comprised $6 \times 30 \mathrm{~m}$ sprints, with a one-minute recovery (including walking time). The 
number of sprints was progressively increased from 6 in training week 1 , to 8 in week 2 , 10 in week 3 and finishing with $12 \times 30 \mathrm{~m}$ sprints in training week 4.

Statistical analyses

Slalom performance and the fastest, slowest, mean and total sprint time during the repeated sprints were analysed using $2 \times 2$ (group $\times$ time) repeated measures ANOVA. An independent samples t-test was used where any interaction effects were observed. T-Tests were used to analyse differences between hockey specific assessments, with significance set at $p<0.05$ a priori, and Cohen's deffect sizes were also calculated for all parameters.

\section{RESULTS}

Analysis of data indicated that there was no significant difference between groups in any performance parameter at baseline. A significant time, group and group by time interaction effect was observed on total, fastest, slowest and mean sprint time $(p<$ 0.05). Follow-up testing indicated a significant improvement in performance in the intervention group for total sprint time (15.2\%), fastest time (12.1\%), slowest time $(14.6 \%)$ and overall average sprint time (15.6\%). There were no significant changes in performance for the control group (Table 1). 
Insert table 1 here

Table 1. Total, fastest, slowest and average time taken to complete the sprint test (All data presented mean $( \pm S D)$ and represented in seconds).

* indicates significant difference from baseline $(p<0.05)$

In the hockey specific assessments, shuttle sprint performance with the stick and stick with ball were significantly improved following the training intervention (stick alone $+2.1 \%$ vs. $-2.8 \%$ performance change $(d=-1.32)$; stick and ball $+0.7 \%$ vs. $-3.8 \%$ performance change $(d=-1.14)$ for the control and treatment groups respectively; figure 2). Slalom sprint performance was not significantly affected either with or without the ball following the treatment intervention ( $d=0.4$ and 0.2 respectively; figure 3 ).

Insert figure 2 here - panel A on the left, panel B on the right

Figure 2. Panel A, shuttle sprint performance; B, sprint performance with stick and ball. ${ }^{*}$ represents a significant difference to baseline 
Insert figure 3 here - panel A on the left, panel B on the right

Figure 3. Panel A, slalom sprint performance; B, slalom performance with stick and ball

\section{DISCUSSION}

While high intensity training (HIT) strategies are commonly investigated in laboratory settings, they often require the use of specialised ergometers and use training protocols that do not replicate team sport specific training approaches well. The purpose of this study was to investigate the effects of 4 -weeks high-intensity training (HIT) on repeated sprint, and hockey related performance. The protocol used specifically mimics the sprint component of many field-based team sports, as indicated by time-motion analyses (27), and although the mechanics of uphill sprinting differ to those of flat sprinting, it has been demonstrated that uphill running results in greater muscle activation $(25,31)$. There were significant improvements in repeated sprint performance, and in changes in hockey related performance measures following 4 weeks of twice weekly uphill run based HIT.

Following 8 sessions of uphill HIT, sprint performance was improved by $12-15 \%$ (Table

1) which is consistent with those observed in laboratory settings (17). However, there is 
a relative paucity of data considering this type of training in more applied settings. Dupont et al. (9) reported that 10 weeks of repeated sprint training in male football players significantly altered $40-\mathrm{m}$ sprint performance (time decreased by $~ 3.5 \%$ ), and improved aerobic running performance. These findings reflect those of Siegler et al. (24), who reported significant improvements in sprint speed and endurance performance in football players following a 10-week HIT intervention, although it has previously been reported that shorter duration training interventions are beneficial to performance. For example Jakeman et al. (17) reported an increase in power output following only 2 weeks of HIT carried out in triathletes. Likewise, Kavaliauskas et al. (18) demonstrate an increase in strength using short duration uphill sprints. In contrast, some shorter duration intervention studies have failed to observe significant changes in power output. Spencer et al. (26) reported no significant difference in power output during repeated sprint testing following 7-weeks of field hockey based training, consisting of 2 strength sessions, 4 endurance sessions and 1 sprint session per week, in elite hockey players, although absolute total work completed during the repeated sprint test was increased. This suggests that a more traditional model of field hockey conditioning was not as effective at increasing power output. Intensity of exercise is crucial to the magnitude of training adaptation (30), and skeletal muscle adaptation to run training is directly related to the intensity of the run program, with greater adaptations shown at $>80 \% \mathrm{VO}_{2}$ peak (8). It should be noted that the competition level of participants in the current study was semi-professional, rather than the elite participants used by Spencer et al. (26), and therefore the lack of effect in that study may be reflective of a higher general conditioning level. However, Spencer et al. (26) 
used cycle sprint exercise training making it difficult to make direct comparisons. The current study required participants to complete running sprint training on an uphill slope, which has been shown to increase anaerobic capacity through greater skeletal muscle ATP concentration (16), and by using the uphill sprint model, effectively increased resistance and intensity during exercise in comparison with flat sprinting $(25,31)$. The biomechanics of the uphill sprint movement may also be more specific to hockey because of the crouched position used during stick and ball activities, although this was not considered in the current study, and could be addressed in future research.

During short repeated sprint activity, there is a rapid utilisation of available ATP, and anaerobic glycolysis and phosphocreatine (PCr) breakdown contribute mainly to its resynthesis (3). During repeated sprint work, ATP resynthesis is achieved to an increasing level by aerobic metabolism $(11,14)$. While the causes of adaptations to this type of training are still being elucidated, a number of investigations have indicated improved lactate kinetics, changes in the efficiency of aerobic metabolism, faster $\mathrm{VO}_{2}$ kinetics and increased citrate synthase activity and PGC- $1 \alpha$ content $(1,4,15,17)$ may all play a role. Similarly, it has been demonstrated that this training approach can improve sprint speed and muscle power, over relatively short periods (17), such as those experienced during participation in a number of team sports, perhaps in relation to changes in glycolytic enzyme activity (5). This type of training therefore appears to be beneficial to eliciting adaptations in both endurance, and power generating capacity. 
The ability to change direction rapidly when moving at high velocities (agility), is crucial to the effective performance of many team sports, and the need to accelerate and decelerate in a variety of directions requires an added level of neural and mechanical complexity and control in comparison with straight line sprinting. Previous research has suggested that adaptations to repeated sprint training include a neural component (6), which is affected to a greater extent following HIT in comparison with low intensity training (7). In the current study, performance in the shuttle sprint, both with stick and stick and ball was significantly improved by $2.8 \%-3.8 \%$ (Figure 1). Similar findings have been reported in 5-10-5 agility, which has a similar turning pattern to the shuttle test, following 4 weeks of uphill sprints (18). However, slalom shuttle performance, which involves multiple direction changes, was not affected as a result of the training intervention (Figure 2). This lack of effect may be due to the fact that the repeated acceleration and deceleration required for changing direction at high speed, reduced the ability to generate the high ground reaction force in a forward direction, which is a determinant of overall sprint performance $(22,23)$. However, it may also be the case, that the relatively short straight-line sprint distance in the slalom resulted in athletes failing to reach maximal velocity, or that the training stimulus (straight line sprinting) may not have been relevant enough to a performance parameter which involves changing direction, and so failed to show any change in this parameter.

\section{PRACTICAL APPLICATIONS}


These findings suggest that using uphill sprinting as part of a training programme is beneficial for physical performance, in this case in hockey. Strength and conditioning professionals should consider including uphill sprint training in addition to/in place of non-sport specific training sessions, as it can elicit superior performance benefits. The added load associated with uphill sprinting as opposed to flat sprint training is also likely to be a factor which influences changes in performance.

\section{REFERENCES}

1. Bailey, SJ, Wilkerson, DP, DiMenna, FJ, and Jones, AM. Influence of repeated sprint training on pulmonary $\mathrm{O} 2$ uptake and muscle deoxygenation kinetics in humans. J Appl Physiol 106: 1875-1887, 2009.

2. Bishop, D, Girard, O, and Mendez-Villanueva, A. Repeated-sprint ability - part II: Recommendations for training. Sports Med 41(9): 741-756, 2011.

3. Bogdanis, GC, Nevill, ME, Boobis, LH, and Lakomy, HKA. Contribution of phosphocreatine and aerobic. J Appl Physiol 80(3): 876-884, 1996.

4. Burgomaster, KA, Howarth, KR, Phillips, SM, Rakobowchuk, M, MacDonald, MJ, McGee, SL, and Gibala, MJ. Similar metabolic adaptations during exercise after low volume sprint interval training and traditional endurance training in humans. $J$ Physiol 586(1): 151-160, 2008. 
5. Carmeli, E, Moas, M, Lennon, S, and Powers, SK. High intensity exercise increases expression of matrix metolloproteinases in fast skeletal muscle fibres. Exp Physiol 90(4): 613-619, 2005.

6. Creer, AR, Ricard, MD, Conlee, RK, Hoyt, GL, and Parcell, AC. Neural, metabolic, and performance adaptations to four weeks of high intensity sprintinterval training in trained cyclists. Int J Sports Med 25: 92-98, 2004.

7. Deschenes, MR, Maresh, CM, Crivello, JF, Armstrong, LE, Kraemer, WJ, and Covault, J. The effects of exercise training of different intensities on neuromuscular junction morphology, J Neurocytol 22: 603-615, 1993.

8. Dudley, GA, Abraham, WM, and Terjung, RL. Influence of exercise intensity and duration on biochemical adaptations in skeletal muscle. J Appl Physiol, 53(4): 844-850, 1982.

9. Dupont, G, Akakpo, K, and Berthoin, S. The effect of in-season, high-intensity interval training in soccer players. J Strength Cond Res 18(3): 584-589, 2004.

10. Gamble, P. Implications and applications of training specificity for coaches and athletes. Strength Cond J 28(3): 54-58, 2006. 
11. Gaitanos, GC, Williams, C, Boobis, LH, and Brooks, S. Human muscle metabolism during intermittent maximal exercise. J Appl Physiol 75(2): 712-719, 1993.

12. Gibala, MJ, Little, JP, van Essen, M, Wilkin, GP, Burgomaster, KA, Safdar, A, Raha, S, and Tarnopolsky, MA. Short-term sprint interval versus traditional endurance training: similar adaptations in human skeletal muscle and exercise performance. J Physiol 15(575): 901-911, 2006.

13. Gibala, MJ, and McGee, SL. Metabolic adaptations to short-term high-intensity interval training: A little pain for a lot of gain? Exerc Sports Sci Rev 36(2): 58-63, 2008.

14. Glaister, M. Multiple sprint work: Physiological responses, mechanisms of fatigue and the influence of aerobic fitness. Sports Med 35(9): 757-777, 2005.

15. Harmer, AR, McKenna, MJ, Sutton, JR, Snow, RJ, Ruell, PA, Booth, J, Thompson, MW, Mackay, NA, Stathis, GC, Crameri, RM, Carey, MF, and Eager, DM. Skeletal muscle metabolic and ionic adaptations during intense exercise following sprint training in humans. J Appl Physiol 89: 1793-1803, 2000.

16. Houston, ME, and Thomson, JA. The response of endurance-adapted adults to intense anaerobic training. Eur J Appl Physiol 36: 207-213, 1977. 
17. Jakeman, JR, Adamson, S, and Babraj, J. Extremely short duration high-intensity training substantially improves endurance performance in triathletes. Appl Physiol, Nutr Metabol 37: 976-981, 2012.

18. Kavaliauskas, M, Kilvington, R, and Babraj, J. Effects of in-season uphill sprinting on physical characteristics in semi-professional soccer players. J Sports Med Phys Fitness in press, 2015.

19. Laursen, PB, Blanchard, MA, and Jenkins, DG. Acute high-intensity interval training improves Tvent and peak power output in highly trained males. Can J Appl Physiol 27(4): 336-348, 2002.

20. Lemmink, KAPM, Elferink-Gemser, MT, and Visscher, C. Evaluation of the reliability of two field hockey specific sprint and dribble tests in young field hockey players. Br J Sports Med 38: 138-142, 2004.

21. Little, JP, Safdar, A, Bishop, D, Tarnopolsky, MA, and Gibala, MJ. An acute bout of high-intensity interval training increases the nuclear abundance of PGC-1 and activates mitochondrial biogenesis in human skeletal muscle. Am J Physiol: Regul, Integr Comp Physiol 300: R1303-R1310, 2011.

22. Morin, JB, Samozino, P, Bonnefoy, R, Edouard, P, Belli, A. Direct measurement of power during one single sprint on treadmill. J Biomech 43: 1970-1975, 2010. 
23. Morin, JB, Bourdin, M, Edouard, P, Peyrot, N, Samozino, P, and Lacour, JR. Mechanical determinants of 100-m sprint running performance. Eur J Appl Physiol 112: 3921-3930, 2012, 2012.

24. Siegler, J, Gaskill, S, and Ruby, B. Changes evaluated in soccer-specific power endurance either with or without a 10-week, in-season, intermittent, high-intensity training protocol. J Strength Cond Res 17(2): 379-387, 2003.

25. Sloniger, MA, Cureton, KJ, Prior, BM, and Evans, EM. Lower extremity muscle activation during horizontal and uphill running. J Appl Physiol, 83(6): 2073-2079, 1997.

26. Spencer, M, Bishop, D, and Lawrence, S. Longitudinal assessment of the effects of field-hockey training on repeated sprint ability. J Sci Med Sport 7(3): 323-334, 2004a.

27. Spencer, M, Lawrence, S, Rechichi, C, Bishop, D, Dawson, B, and Goodman, C. Time-motion analysis of elite field hockey, with special reference to repeatedsprint activity. J Sports Sci, 22: 843-850, 2004b. 
28. Sporis, G, Ruzic, L, and Leko, G. The anaerobic endurance of elite soccer players improved after a high-intensity training intervention in the 8-week conditioning program. J Strength Cond Res, 22(2): 559-566, 2008.

29. Stone, NM, and Kilding, AE. Aerobic conditioning for team sport athletes. Sports Med 39(8): 615-642, 2009.

30. Wenger, HA, and Bell, GJ. The interactions of intensity, frequency and duration of exercise training in altering cardiorespiratory fitness. Sports Med 3: 346-356, 1986.

31. Yokozawa, T, Fujii, N, and Ae, M. (2007). Muscle activities of the lower limb during level and uphill running. J Biomech, 40: 3467-3475, 2007. 


\begin{tabular}{|c|c|c|c|c|}
\hline & CONDITION & PRE & POST & $d$ \\
\hline \multirow{4}{*}{$\begin{array}{l}\text { Total sprint } \\
\text { time (s) }\end{array}$} & Control & $25.94(2.8)$ & $25.23(2.8)$ & \multirow{4}{*}{-0.95} \\
\hline & $95 \% \mathrm{Cl}$ & 20.4-31.4 & $19.8-30.6$ & \\
\hline & Treatment & $27.26(1.0)$ & $23.14(1.4)^{*}$ & \\
\hline & $95 \% \mathrm{Cl}$ & 25.3-29.1 & 20.3-25.9 & \\
\hline \multirow{4}{*}{$\begin{array}{l}\text { Fastest sprint } \\
\text { time (s) }\end{array}$} & Control & $4.08(0.5)$ & $4.1(0.5)$ & \multirow{4}{*}{-1.08} \\
\hline & $95 \% \mathrm{Cl}$ & $3.2-5.0$ & $3.1-5.0$ & \\
\hline & Treatment & $4.21(0.3)$ & $3.7(0.4)^{*}$ & \\
\hline & $95 \% \mathrm{Cl}$ & $3.6-4.8$ & $2.9-4.3$ & \\
\hline \multirow{4}{*}{$\begin{array}{l}\text { Slowest sprint } \\
\text { time (s) }\end{array}$} & Control & $4.5(0.5)$ & $4.3(0.4)$ & \multirow{4}{*}{-0.43} \\
\hline & $95 \% \mathrm{Cl}$ & 3.6-5.4 & $3.4-5.1$ & \\
\hline & Treatment & $4.8(0.2)$ & $4.1(0.3)^{*}$ & \\
\hline & $95 \% \mathrm{Cl}$ & $4.4-5.2$ & $3.6-4.7$ & \\
\hline \multirow{4}{*}{$\begin{array}{l}\text { Mean sprint } \\
\text { time (s) }\end{array}$} & Control & $4.3(0.5)$ & $4.2(0.5)$ & \multirow{4}{*}{-0.95} \\
\hline & $95 \% \mathrm{Cl}$ & $3.4-5.2$ & $3.3-5.1$ & \\
\hline & Treatment & $4.5(0.2)$ & $3.9(0.2)^{*}$ & \\
\hline & $95 \% \mathrm{Cl}$ & $4.2-4.8$ & $3.4-4.3$ & \\
\hline
\end{tabular}

Table 1. Total, fastest, slowest and average time taken to complete the sprint test (All data presented mean ( $\pm S D$ ) and represented in seconds), 95\% confidence intervals and Cohen's $d$ value for effect size

* indicates significant difference from baseline $(p<0.05)$ 


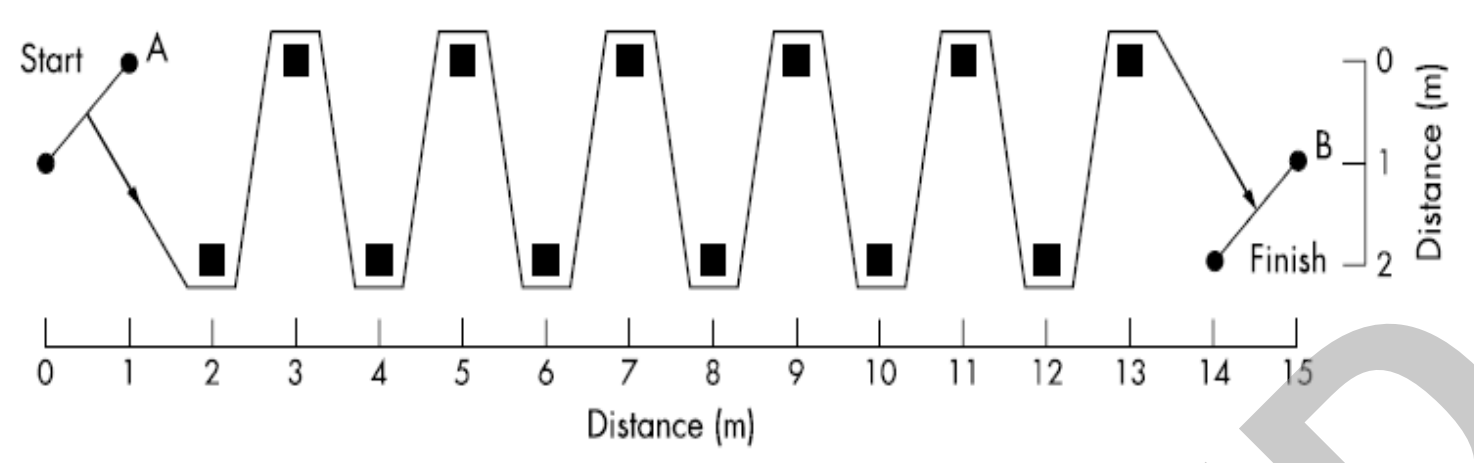

Figure 1- Slalom sprint test and dribble test (Slalom STD). 
A

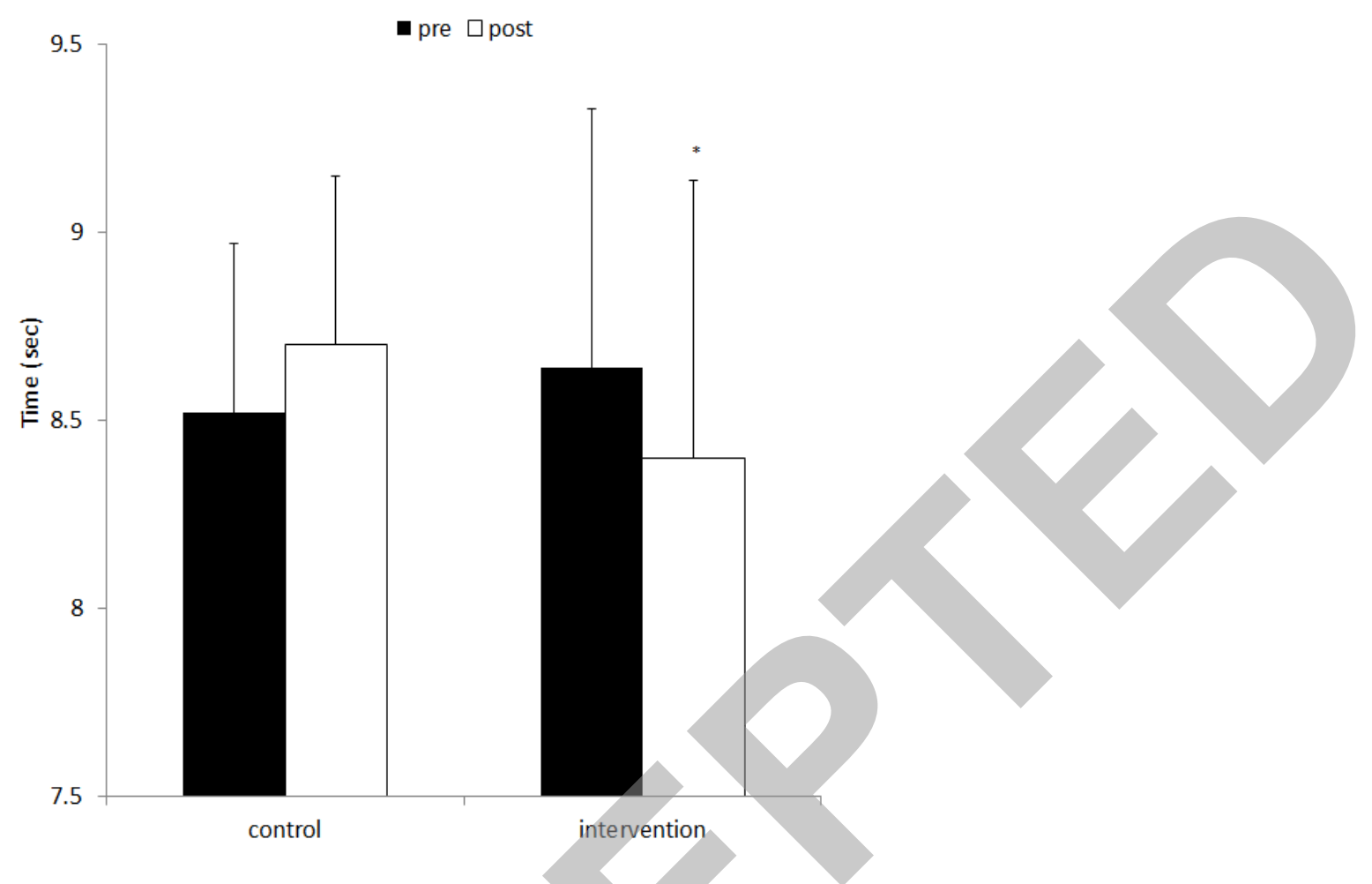

B

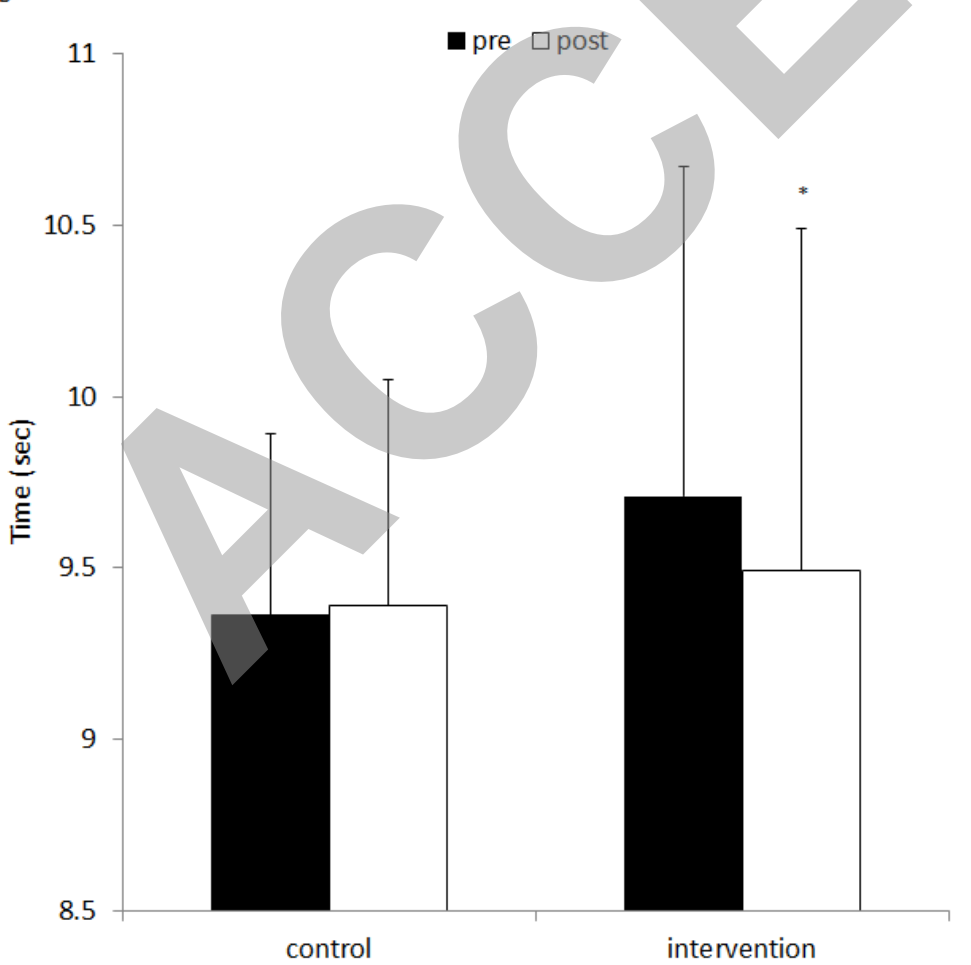


Figure 2. Panel A, shuttle sprint performance; B, sprint performance with stick and ball. *represents a significant difference to baseline 
A

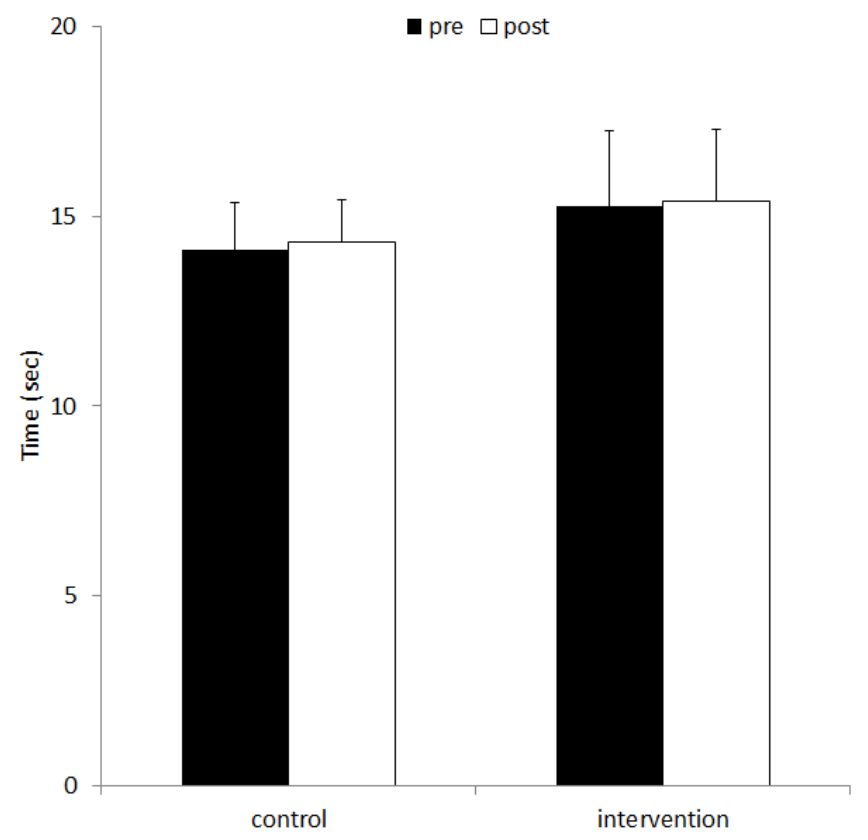

B

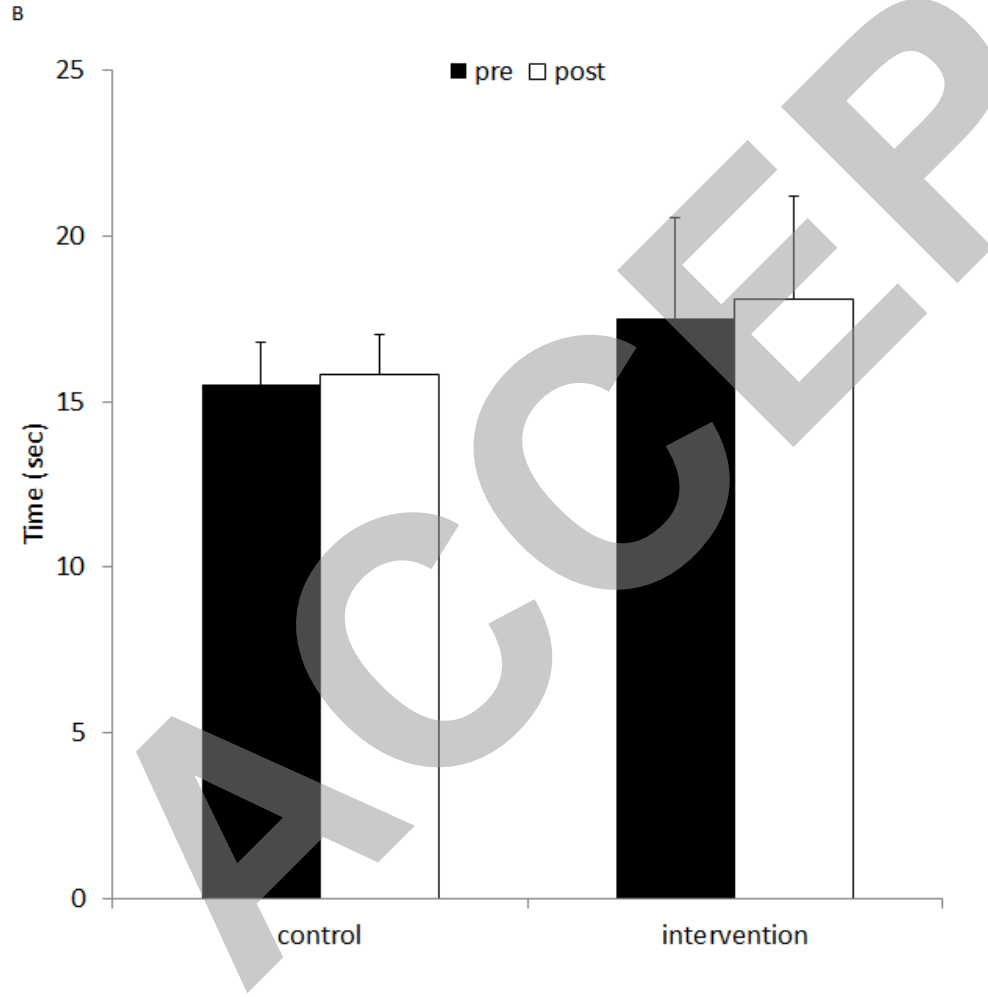

Figure 3. Panel A, slalom sprint performance; B, slalom performance with stick and ball 